\title{
Associations between depressive symptoms and geriatric syndromes in community-dwelling older adults in Japan: a cross-sectional study
}

Masakazu Imaoka ( $\sim$ imaokamasakazu@gmail.com )

Osaka Kawasaki Rehabilitation Univercity https://orcid.org/0000-0002-5849-7107

Hidetoshi Nakao

Osaka Kawasaki Rehabilitation University

Misa Nakamura

Osaka Kawasaki Rehabilitation University

Fumie Tazaki

Osaka Kawasaki Rehabilitation University

Mitsumasa Hida

Osaka Kawasaki Rehabilitation University

Tomoko Omizu

Kansai University fo Welfare Sciences

\section{Ryota Imai}

Osaka Kawasaki Rehabilitation University

\section{Masatoshi Takeda}

Osaka Kawasaki Rehabilitation University

\section{Research article}

Keywords: depressive symptoms, frailty, sarcopenia, locomotive syndrome, elderly, Japan

Posted Date: September 21st, 2020

DOI: https://doi.org/10.21203/rs.3.rs-63598/v1

License: (1) (1) This work is licensed under a Creative Commons Attribution 4.0 International License. Read Full License

Version of Record: A version of this preprint was published at Preventive Medicine Reports on June 1st, 2021. See the published version at https://doi.org/10.1016/j.pmedr.2021.101353. 


\section{Abstract \\ Background}

It is estimated that $7.2 \%$ of community-dwelling older adults in worldwide have major depression. This cross-sectional study aimed to investigate the relationship between geriatric syndromes and depressive symptoms.

\section{Methods}

Data were obtained from 363 older adults (mean age $73.6 \pm 6.6$ years; women $=75.8 \%$ ) who participated in a community-based health check. Depressive symptoms were assessed using the 15-item Geriatric Depression Scale (GDS-15). Depression was defined as a GDS-15 score of $\geq 5$. Further, geriatric syndromes in participants-such as frailty, sarcopenia, and locomotive syndrome-were assessed.

\section{Results}

There was a $28.1 \%$ prevalence of depressive symptoms. In a logistic regression analysis with depressive symptoms as the dependent variable, both pre-frailty (odds ratio [OR] 1.85, 95\% confidence interval [Cl] $1.09-3.01$ ) and frailty (OR $5.45,95 \% \mathrm{Cl} 2.23-13.31$ ) were found to be significantly higher in the depressive group.

\section{Conclusions}

Our findings suggest that depressive symptoms are associated with frailty and pre-frailty in communitydwelling older adults in Japan.

\section{Background}

Depression is a common psychiatric disorder that affects approximately 322 million people worldwide [1]. It is estimated that $7.2 \%$ of community-dwelling older adults in worldwide have major depression, and $17.1 \%$ had depression throughout their lives [2]. Factors associated with depression in old age include deterioration of life functions [3] and decline in physical [4,5] and cognitive functions [6]. Other factors related to living conditions, such as living alone and being divorced, have also been reported [7].

Depressive symptoms are associated with adverse health outcomes, including impairment [8], mortality [9], and poor quality of life [10].

Previous studies with community-dwelling older adults have reported associations between depressive symptoms and physical $[11,12]$ and cognitive functions [13]. In the 3-year prospective cohort study with 680 community-dwelling older adults by Yoshida et al. [12], continued physical activity in old age was the 
only factor associated with a reduction in the incidence of depressive symptoms. A previous 14-year prospective study in 1,417 older adults with no chronic illness found that exercise habits were significantly associated with depression [11]. Another study identified depression as a risk factor for dementia [13]. To date, depressive symptoms have been evaluated from the perspective of exercise or cognitive function alone, but not both.

Recent geriatric syndrome studies addressed a number of complex conditions associated with aging, such as depression, frailty [14], sarcopenia [15], and locomotive syndrome [16], while several studies focused on motor functions [17-21]. One study reported that community-dwelling frail older adults are at high risk of depression [22]. A systematic review reported an independent association between depression and sarcopenia in community-dwelling older adults [23-25], and a cross-sectional study reported an association between depression and locomotive syndrome [16, 26]. Although the association between these geriatric syndromes and depression is clear, the strength of the association is not evident.

Therefore, we conducted a cross-sectional study of typical geriatric syndromes-including frailty, sarcopenia, and locomotive syndrome-to examine their association with depression. Our findings may contribute to the prioritization of the clinical evaluation of geriatric syndromes and highlight the importance of focusing on the items included in the geriatric syndromes, such as muscle strength, muscle mass, mobility, fatigue, and weight loss.

\section{Methods}

\section{Aim, design, and setting}

\section{Study population}

Data from the Kaizuka Dementia Prevention Study 2018 and 2019 were used in this cross-sectional study. The Kaizuka Study was a community-based health check conducted in collaboration with the Osaka Kawasaki Rehabilitation University (Kaizuka City Office) and Cognitive Reserve Research Center in Osaka, Japan.

The inclusion criteria for participation were being residents of Kaizuka City and aged $\geq 60$ years at the time of the study. For recruitment, a leaflet was inserted in a newspaper and posted at the public hall, and interested participants applied telephonically. Exclusion criteria were having a history of depression ( $n=$ $13)$, being aged 60 or under $(n=25)$, and missing data on the survey $(n=7)$. Finally, data from 363 participants (mean age 73.6 [SD \pm 6.6$]$ years; women $n=275$ [75.6\%]) were analyzed (Fig. 1).

\section{Ethical considerations}

This study was approved by the Ethics Committee of the authors' affiliate institution. Written informed consent was obtained from all participants in accordance with the tenets of the Declaration of Helsinki. 
No compensation was provided for participation. This study was approved by the Ethics Committee of the Osaka Kawasaki Rehabilitation University (Reference No. OKRU29-A016).

\section{Measures}

\section{Depressive symptoms}

We used the Japanese version of the Geriatric Depression Scale 15 (GDS-15) to determine the existence of depressive symptoms among the participants. The GDS-15 is a self-report instrument comprising 15 items that assess the individual's mood $[27,28]$. A cut-off point of $4 / 5$ was used to define depressive symptoms in this study.

\section{Body muscle mass}

We collected data on physiological parameters obtained using bioelectrical impedance analysis (InBody270; InBody, Tokyo, Japan) at 20 and $1000 \mathrm{kHz}$ frequencies from participants' electronic medical records [29]. Measurements were recorded within 30 seconds by the staff. Body mass index (BMI) was calculated by dividing body weight $(\mathrm{kg})$ by squared height $\left(\mathrm{m}^{2}\right)$. The appendicular skeletal muscle index $(\mathrm{SMI})$ was derived from the appendicular muscle mass $(\mathrm{kg})$ divided by squared height $\left(\mathrm{m}^{2}\right)$. Low muscle mass was defined as a height-adjusted skeletal muscle mass $<7.0 \mathrm{~kg} / \mathrm{m}^{2}$ for men and $<5.7 \mathrm{~kg} / \mathrm{m}^{2}$ for women.

\section{Muscle strength}

Muscle strength was measured by handgrip strength, which has been reported to be significantly associated with whole-body muscle strength [30]. The maximum voluntary isometric strength of the handgrip was measured using a Grip-D hand dynamometer (Takei; Niigata, Japan) for the dominant hand while in a standing position. Low muscle strength was defined as a handgrip strength $<26 \mathrm{~kg}$ in men and $<18 \mathrm{~kg}$ in women.

\section{Gait speed}

We instructed participants to walk $6.4 \mathrm{~m}$ (divided into two 2-m zones at each end and a 2.4-m zone in the middle) at a speed they found comfortable. The time needed (s) to pass the 2.4-m middle zone was measured to calculate the gait speed $(\mathrm{m} / \mathrm{s})$ [31]. Participants could use a cane or walker if they were unable to walk without help. We used the average of five gait trials.

\section{Cognitive status}

We used the Japanese version of the Mini-Mental State Examination (MMSE), which is widely used to assess cognitive function and screen for cognitive impairment [23,32]. MMSE scores range from 0 to 30 , with lower scores representing weaker cognitive function.

\section{Locomotive syndrome}


We measured locomotive syndrome using the two-step test. Participants were asked to take two steps forward as far as possible and stop after the second step [33]. Participants were retested if they lost balance during the test or if they did not stop after the second step. They were not allowed to jump. The test was carried out until two results were obtained. The maximum length $(\mathrm{m})$ covered in the two tests was selected and divided by the participant's height $(\mathrm{m})$. Results were categorized as robust $(\geq 1.3 \mathrm{~m} / \mathrm{m})$, Locomo1 $(<1.3 \mathrm{~m} / \mathrm{m})$, and Locomo2 $(<1.1 \mathrm{~m} / \mathrm{m})$, based on the Japanese Orthopedic Association criteria [16].

\section{Sarcopenia}

We determined participants' sarcopenia status using the Asian Working Group for Sarcopenia (AWGS) consensus recommendations [15] in terms of the results of the gait test, muscle strength, and muscle mass. Participants with skeletal muscle mass loss (SMI $\left[<7.0 \mathrm{~kg} / \mathrm{m}^{2}\right.$ for men and $<5.7 \mathrm{~kg} / \mathrm{m}^{2}$ for women]), low physical function (low grip strength [ $<26 \mathrm{~kg}$ for men and $<18 \mathrm{~kg}$ for women] and slowness, indicated by normal walking speed $[<0.8 \mathrm{~m} / \mathrm{sec}]$ ), and skeletal muscle mass loss without low physical function were determined as having pre-sarcopenia.

\section{Frailty}

The definition of frailty used in this study [14] has been described in detail elsewhere. The components of frailty, as defined by Fried et al. [14], are as follows: weight loss, weakness, exhaustion, low activity level, and slowness. Weight loss was determined using the question "Have you lost $2 \mathrm{~kg}$ or more in the past 6 months?" taken from the Kihon Checklist, a self-report, comprehensive health checklist. Exhaustion was determined based on a response of "yes" to another question from the Kihon Checklist, "In the last 2 weeks, have you felt tired for no reason?" [34]. Weakness was defined as low muscle strength based on the grip strength assessment described earlier. Low activity level was defined as a negative response to the following questions: "Do you engage in moderate levels of physical exercise or sports aimed at health?" and "Do you engage in low levels of physical exercise aimed at health?" Slowness was defined as a walking speed of $<1.0 \mathrm{~m} / \mathrm{s}$. Participants were classified as "robust" if they had none of these symptoms, "pre-frail" if they had one or two symptoms, and "frail" in case of three or more symptoms [35].

\section{Covariates}

We collected data on sociodemographic variables, including age (years) and gender. The covariates in this study comprised these sociodemographic variables, as well as cognitive function and physical function.

\section{Statistical analysis}

All analyses were performed using IBM SPSS Statistics version 24.0 (IBM Corp., Armonk, NY, USA). Pvalues $<0.05$ were considered to be statistically significant. To compare the depressive and nondepressive groups, we used Pearson's $\chi^{2}$ tests for categorical variables, Student's $t$-test for continuous variables, and Mann-Whitney's U-test for ordinal variables. Non-linear logistic regression analyses were 
performed to examine the association between sarcopenia, frailty, locomotive syndrome, and depressive symptoms. There were two regression models: one using crude odds ratios (ORs), while the other using adjusted ORs. Presence of depressive symptoms was the dependent variable in both models.

\section{Results}

The participant characteristics are shown in Table 1. Of the 363 participants, 102 (28.1\%) had some depressive symptoms or a specific category of depressive symptoms, as determined by their GDS-15 scores. Participants in the depressive group were more likely to be frail or pre-frail and had lower MMSE scores and a higher frailty rate than those in the non-depressive group.

Table 2 shows the results of the univariate and multivariate logistic regression analyses. On univariate logistic regression analysis, pre-frailty (OR 1.81, 95\% confidence interval $[\mathrm{Cl}] 1.09-3.01)$ and frailty $(\mathrm{OR}$ $5.55,95 \% \mathrm{Cl} 2.31-13.30$ ) were significantly related to depressive symptoms (Crude Model). After multivariate adjusting for potential covariates, pre-frailty (OR 1.85, 95\% $\mathrm{Cl} 1.09-3.01$ ) and frailty (OR 5.45, $95 \% \mathrm{Cl} 2.23-13.31$ ) were found to be significantly related to depressive symptoms (Adjusted Model 1).

\section{Discussion}

This cross-sectional study showed that pre-frailty and frailty were significantly associated with depressive symptoms in older adults after adjusting for potential covariates. Thus, our results suggest that depressive symptoms are associated with pre-frailty and frailty as defined by the JapanCardiovascular Health Study (J-CHS). However, other geriatric syndrome, sarcopenia, locomotive syndrome were not associated with depressive symptoms.

Previous studies have reported a prevalence of depression among older adults ranging from $17.8-27.2 \%$ [36-38]. In this study, the prevalence of depressive symptoms was $28.1 \%$, which is relatively high. It is possible that, because the participants in this study were recruited via public hall postings, city hall notices, and newspaper leaflets, the participation rate of older adults with health anxiety was higher. In a study by Tsujiguchi et al. [38] among 2,470 Japanese older adults selected from the same population, the prevalence of depression was reported to be $27.2 \%$. The results of the present study might be generalizable to other Japanese older adults, given the similar prevalence rates.

The recent interest in "overlapping comorbidities," specifically, frailty and depression, has given rise to reviews indicating a positive association between the two [39-41]. A study with 958 adults older than 60 years found that depressive symptoms had a significant association with frailty (OR 1.8), which supports our results [42]. Another longitudinal study reported that frailty is predictive of future depressive symptoms [43].

Previous studies on frailty and depression as overlapping geriatric syndromes reported that the latent factors of frailty that were significantly correlated with depression were biological syndrome $(\rho=0.68, p<$ 0.01), functional domains $(\rho=0.70, p<0.01)$, and frailty index $(\rho=0.61, p<0.01)$ [44]. The biological 
syndrome model of frailty was operationalized in terms of five criteria proposed by Fried and colleagues [14], who analyzed data from the Cardiovascular Health Study. The frailty index is a count of 70 clinical deficits, including presence of diseases, difficulty in performing daily activities, and other physical and neurological signs and symptoms. In terms of functional domains, frailty was defined as functional impairment in at least two of four domains: physical, nutritive, cognitive, and sensory. For example, a previous study [45] reported that models of frailty and depression with differing numbers of classes that represent the severity of the syndromes (two in frailty; three in depression) provided a better fit to their data, although the constructs shared a substantial amount of variance (latent kappa coefficient $=0.66$ ); moreover, these also helped identify overlapping subgroups of participants. Using confirmatory factor analysis in the Health and Retirement Study among 3,453 participants, a moderate correlation (0.68) was found between latent categorical models of depression and the "biological definition" of frailty [46]. These findings suggest that pre-frailty and frailty are associated with depressive symptoms. Frailty comprises motor functions such as walking speed and grip strength, and these can be improved by exercise therapy, even in older adults [47]. Therefore, it may be possible to use non-pharmacological interventions to reduce depression in older adults.

Contrary to expectations, no geriatric syndromes other than frailty were associated with depressive symptoms. Although locomotive syndrome is an independent risk factor for depression [16, 26], in this study we found no significant difference between the non-depressive and depressive groups. However, an earlier study based on the 25-item Geriatric Locomotive Function Scale (GLFS-25) reported a correlation between depressive symptoms and locomotive syndrome among 224 community-dwelling older adults [26]. The reason for this discrepancy may be that the GLFS-25 questionnaire includes items on psychological characteristics, such as pain and anxiety, which may be better measures of depressive symptoms.

Previous studies of sarcopenia [23-25] found a correlation between sarcopenia and depression; however, the present study did not find a significant correlation between the two. In a 6-year prospective study with 1,731 community-dwelling older adults, sarcopenia alone was not associated with depression, but was associated with sarcopenia obesity [48]. Therefore, sarcopenia, which indicates muscle wastage, is not associated with depression alone, but may be associated with depression with regard to BMI. Speed et al. [49] investigated the relationship between fat weight, non-fat weight, and depression by analyzing the body composition data of 800,000 people and reported that fat weight was a risk factor for depression. The aforementioned findings suggest that it is important to consider sarcopenia obesity and obesity, rather than sarcopenia alone, when assessing the risk of depression.

Our study had some limitations. First, we used screening tools rather than full diagnostic procedures to assess depressive symptoms. Second, the cross-sectional design of the study precluded the assumption of a causal relationship between depressive symptoms and frailty. Third, study data were obtained from a single city, which limits the generalizability of the results, as meaningful activities in daily life are also affected by geographic characteristics. Finally, the 363 older adults enrolled represented approximately 
$3 \%$ of older adults living in urban areas and were not randomly selected. Therefore, sampling bias cannot be ruled out.

\section{Conclusion}

This study found a significant association of pre-frailty and frailty with depressive symptoms among community-dwelling older adults. Among the geriatric syndromes considered, frailty was strongly related to depressive symptoms, suggesting that it is possible to prevent depression by reducing frailty. We believe that the present study may be useful in developing measures to reduce the risk of future depressive symptoms among older adults.

\section{Abbreviations}

GDS-15: Geriatric Depression Scale-15, BMI Body:mass index, SMI:Skeletal muscle index, MMSE:MiniMental State Examination, AWGS:Asian Working Group for Sarcopenia

\section{Declarations}

\section{Ethics approval and consent to participate}

This study was approved by the Ethics Committee of the authors' affiliate institution. Written informed consent was obtained from all participants in accordance with the tenets of the Declaration of Helsinki.

\section{Consent for publication}

Not applicable.

\section{Availability of data and materials}

The data that support the findings of this study are available from the Kaizuka Dementia Prevention Study Project Team; however, restrictions apply to the availability of these data, which were used under license for the current study, and are therefore not publicly available. Data are, however, available from the authors upon reasonable request and with permission from the Kaizuka Dementia Prevention Study Project Team.

\section{Competing interests}

The authors declare that they have no competing interests.

\section{Funding}

Not applicable 


\section{Authors' contributions}

$\mathrm{HN}, \mathrm{FT}, \mathrm{MH}, \mathrm{TO}$, and RI collected data, organized the dataset, and provided advice for consideration. MN contributed to the planning of research plans and survey items. TM organized the entire study. All authors read and approved the final manuscript.

\section{Acknowledgements}

We would like to thank the individuals who participated in data collection and data entry, as well as the Kaizuka Municipal Office for their valuable support and participation.

\section{References}

1. World Health Organization. Depression and other common mental disorders global health estimates. Geneva: WHO; 2017. https://www.who.int/mental_health/management/depression/. prevalence_global_health_estimates/en/. Accessed 10 Aug 2020.

2. Luppa M, Sikorski C, Luck T, Ehreke L, Konnopka A, Wiese B, et al. Age- and gender-specific prevalence of depression in latest-life-systematic review and meta-analysis. J Affect Disord. 2012;136:212-21.

3. Iwasa H, Yoshida Y, Kumagai S, Ihara K, Yoshida H, Suzuki T. Depression status as a reliable predictor of functional decline among Japanese community-dwelling older adults: a 12-year population-based prospective cohort study. Int J Geriatr Psychiatry. 2009;24:1192-200.

4. Hu L, Smith L, Imm KR, Jackson SE, Yang L. Physical activity modifies the association between depression and cognitive function in older adults. J Affect Disord. 2019;246:800-5.

5. Penninx BW, Guralnik JM, Ferrucci L, Simonsick EM, Deeg DJ, Wallace RB. Depressive symptoms and physical decline in community-dwelling older persons. JAMA. 1998;279:1720-6.

6. Potvin $\mathrm{O}$, Forget $\mathrm{H}$, Grenier S, Preville M, Hudon C. Anxiety, depression, and 1-year incident cognitive impairment in community-dwelling older adults. J Am Geriatr Soc. 2011;59:1421-8.

7. Weyerer S, Eifflaender-Gorfer S, Kohler L, Jessen F, Maier W, Fuchs A, et al. Prevalence and risk factors for depression in non-demented primary care attenders aged 75 years and older. J Affect Disord. 2008;111:153-63.

8. Heser K, Stein J, Luppa M, Wiese B, Mamone S, Weyerer S, et al. Late-life depressive symptoms are associated with functional impairment cross-sectionally and over time: results of the AgeMooDe Study. J Gerontol B Psychol Sci Soc Sci. 2020;75:811-20.

9. Murphy RA, Hagaman AK, Reinders I, Steeves JA, Newman AB, Rubin SM, et al. Depressive trajectories and risk of disability and mortality in older adults: longitudinal findings from the health, aging, and body composition study. J Gerontol A Biol Sci Med Sci. 2016;71:228-35. 
10. Chachamovich E, Fleck M, Laidlaw K, Power M. Impact of major depression and subsyndromal symptoms on quality of life and attitudes toward aging in an international sample of older adults. Gerontologist. 2008;48:593-602.

11. Smith TL, Masaki KH, Fong K, Abbott RD, Ross GW, Petrovitch H, et al. Effect of walking distance on 8-year incident depressive symptoms in elderly men with and without chronic disease: the HonoluluAsia Aging Study. J Am Geriatr Soc. 2010;58:1447-52.

12. Yoshida Y, Iwasa H, Kumagai S, Suzuki T, Awata S, Yoshida H. Longitudinal association between habitual physical activity and depressive symptoms in older people. Psychiatry Clin Neurosci. 2015;69:686-92.

13. Livingston G, Sommerlad A, Orgeta V, Costafreda SG, Huntley J, Ames D, et al. Dementia prevention, intervention, and care. Lancet. 2017;390:2673-734.

14. Fried LP, Tangen CM, Walston J, Newman AB, Hirsch C, Gottdiener J, et al. Frailty in older adults: evidence for a phenotype. J Gerontol A Biol Sci Med Sci. 2001;56:M146-56.

15. Chen LK, Liu LK, Woo J, Assantachai P, Auyeung TW, Bahyah KS, et al. Sarcopenia in Asia: consensus report of the Asian Working Group for Sarcopenia. J Am Med Dir Assoc. 2014;15:95-101.

16. Ikemoto T, Inoue M, Nakata M, Miyagawa H, Shimo K, Wakabayashi T, et al. Locomotive syndrome is associated not only with physical capacity but also degree of depression. J Orthop Sci. 2016;21:361-5.

17. Kojima G, lliffe S, Walters K. Frailty index as a predictor of mortality: a systematic review and metaanalysis. Age Ageing. 2018;47:193-200.

18. Liu P, Hao Q, Hai S, Wang H, Cao L, Dong B. Sarcopenia as a predictor of all-cause mortality among community-dwelling older people: a systematic review and meta-analysis. Maturitas. 2017;103:1622.

19. Matsumoto $H$, Hagino $H$, Wada T, Kobayashi E. Locomotive syndrome presents a risk for falls and fractures in the elderly Japanese population. Osteoporos Sarcopenia. 2016;2:156-63.

20. Vermeiren S, Vella-Azzopardi R, Beckwee D, Habbig AK, Scafoglieri A, Jansen B, et al. Frailty and the prediction of negative health outcomes: a meta-analysis. J Am Med Dir Assoc. 2016;17(2):1163. .e11163.e17.

21. Woo J, Leung J, Morley JE. Defining sarcopenia in terms of incident adverse outcomes. J Am Med Dir Assoc. 2015;16:247-52.

22. Makizako H, Shimada H, Doi T, Yoshida D, Anan Y, Tsutsumimoto K, et al. Physical frailty predicts incident depressive symptoms in elderly people: prospective findings from the Obu Study of Health Promotion for the Elderly. J Am Med Dir Assoc. 2015;16:194-9.

23. Chang KV, Hsu TH, Wu WT, Huang KC, Han DS. Is sarcopenia associated with depression? A systematic review and meta-analysis of observational studies. Age Ageing. 2017;46:738-46.

24. Hayashi T, Umegaki H, Makino T, Cheng XW, Shimada H, Kuzuya M. Association between sarcopenia and depressive mood in urban-dwelling older adults: a cross-sectional study. Geriatr Gerontol Int. 2019;19(6):508-12. 
25. Lee I, Cho J, Hong H, Jin Y, Kim D, Kang H. Sarcopenia is associated with cognitive impairment and depression in elderly Korean women. Iran J Public Health. 2018;47:327-34.

26. Nakamura M, Hashizume H, Nomura S, Kono R, Utsunomiya H. The relationship between locomotive syndrome and depression in community-dwelling elderly people. Curr Gerontol Geriatr Res. 2017;2017:4104802.

27. Burke WJ, Roccaforte WH, Wengel SP. The short form of the Geriatric Depression Scale: a comparison with the 30-item form. J Geriatr Psych Neur. 1991;4:173-8.

28. Sugishita K, Sugishita M, Hemmi I, Asada T, Tanigawa T. A validity and reliability study of the Japanese version of the Geriatric Depression Scale 15 (GDS-15-J). Clin Gerontol. 2017;40:233-40.

29. Nakamura M, Tazaki F, Nomura K, Takano T, Hashimoto M, Hashizume H, et al. Cognitive impairment associated with locomotive syndrome in community-dwelling elderly women in Japan. Clin Interv Aging. 2017;12:1451-7.

30. Makizako H, Shimada H, Doi T, Tsutsumimoto K, Lee S, Lee SC, et al. Age-dependent changes in physical performance and body composition in community-dwelling Japanese older adults. J Cachexia Sarcopenia Muscle. 2017;8:607-14.

31. Shimada H, Suzuki T, Suzukawa M, Makizako H, Doi T, Yoshida D, et al. Performance-based assessments and demand for personal care in older Japanese people: a cross-sectional study. BMJ Open. 2013;3:e002424.

32. Folstein MF, Folstein SE, McHugh PR. "Mini-mental state". A practical method for grading the cognitive state of patients for the clinician. J Psychiatr Res. 1975;12:189-98.

33. Ogata T, Muranaga S, Ishibashi H, Ohe T, Izumida R, Yoshimura N, et al. Development of a screening program to assess motor function in the adult population: a cross-sectional observational study. J Orthop Sci. 2015;20:888-95.

34. Fukutomi E, Okumiya K, Wada T, Sakamoto R, Ishimoto Y, Kimura Y, et al. Relationships between each category of 25-item frailty risk assessment (Kihon Checklist) and newly certified older adults under Long-Term Care Insurance: a 24-month follow-up study in a rural community in Japan. Geriatr Gerontol Int. 2015;15:864-71.

35. Shimada H, Makizako H, Lee S, Doi T, Lee S, Tsutsumimoto K, et al. Impact of cognitive frailty on daily activities in older persons. J Nutr Health Aging. 2016;20:729-35.

36. Li D, Zhang DJ, Shao JJ, Qi XD, Tian L. A meta-analysis of the prevalence of depressive symptoms in Chinese older adults. Arch Gerontol Geriatr. 2014;58:1-9.

37. Maruta M, Makizako H, Ikeda Y, Miyata H, Nakamura A, Han G, et al. Associations between depressive symptoms and satisfaction with meaningful activities in community-dwelling Japanese older adults. J Clin Med. 2020;9:795.

38. Tsujiguchi H, Thi Thu Nguyen T, Goto D, Miyagi S, Kambayashi Y, Hara A, et al. Relationship between the intake of n-3 polyunsaturated fatty acids and depressive symptoms in elderly Japanese people: differences according to sex and weight status. Nutrients. 2019;11:775. 
39. Buigues C, Padilla-Sanchez C, Garrido JF, Navarro-Martinez R, Ruiz-Ros V, Cauli O. The relationship between depression and frailty syndrome: a systematic review. Aging Ment Health. 2015;19:762-72.

40. Mezuk B, Edwards L, Lohman M, Choi M, Lapane K. Depression and frailty in later life: a synthetic review. Int J Geriatr Psychiatry. 2012;27:879-92.

41. Vaughan L, Corbin AL, Goveas JS. Depression and frailty in later life: a systematic review. Clin Interv Aging. 2015;10:1947-58.

42. Pegorari MS, Tavares DM. Factors associated with the frailty syndrome in elderly individuals living in the urban area. Rev Lat Am Enfermagem. 2014;22:874-82.

43. Feng L, Nyunt MS, Feng L, Yap KB, Ng TP. Frailty predicts new and persistent depressive symptoms among community-dwelling older adults: findings from Singapore longitudinal aging study. J Am Med Dir Assoc. 2014;15:76.e7-76.e12.

44. Lohman M, Dumenci L, Mezuk B. Depression and frailty in late life: evidence for a common vulnerability. J Gerontol B Psychol Sci Soc Sci. 2016;71:630-40.

45. Mezuk B, Lohman M, Dumenci L, Lapane KL. Are depression and frailty overlapping syndromes in mid- and late-life? A latent variable analysis. Am J Geriatr Psychiatry. 2013;21:560-9.

46. Lohman M, Dumenci L, Mezuk B. Sex differences in the construct overlap of frailty and depression: evidence from the Health and Retirement Study. J Am Geriatr Soc. 2014;62:500-5.

47. Chan DC, Tsou HH, Yang RS, Tsauo JY, Chen CY, Hsiung CA. A pilot randomized controlled trial to improve geriatric frailty. BMC Geriatrics. 2012;12:58.

48. Ishii S, Chang C, Tanaka T, Kuroda A, Tsuji T, Akishita M. The association between sarcopenic obesity and depressive symptoms in older Japanese adults. PLoS One. 2016;11:e0162898.

49. Speed MS, Jefsen OH, Børglum AD, Speed D, Østergaard SD. Investigating the association between body fat and depression via Mendelian randomization. Transl Psychiatry. 2019;9:1-9.

\section{Tables}




\begin{tabular}{|c|c|c|c|c|c|c|c|c|c|c|}
\hline \multirow{3}{*}{$\begin{array}{l}\text { Variable } \\
\text { Age, years }\end{array}$} & \multicolumn{3}{|c|}{$\begin{array}{l}\text { All participants } \\
\quad(n=363)\end{array}$} & \multicolumn{3}{|c|}{$\begin{array}{l}\text { Non-depressive group } \\
\qquad(n=261)\end{array}$} & \multicolumn{3}{|c|}{$\begin{array}{c}\text { Depressive group } \\
(n=102)\end{array}$} & \multirow[b]{2}{*}{$p$-value } \\
\hline & \multicolumn{3}{|c|}{ mean $\pm \mathrm{SD}$} & \multicolumn{3}{|c|}{ mean $\pm \mathrm{SD}$} & \multicolumn{3}{|c|}{ mean $\pm \mathrm{SD}$} & \\
\hline & 73.6 & \pm & 6.6 & 73.4 & \pm & 6.5 & 74.1 & \pm & 6.8 & 0.33 \\
\hline Female sex, $n(\%)$ & 275 & (75.8) & & 197 & $(75.5)$ & & 76 & $(74.5)$ & & 0.89 \\
\hline Grip strength, $\mathrm{kg}$ & 23.9 & \pm & 6.9 & 24.1 & \pm & 6.9 & 23.5 & \pm & 7.0 & 0.43 \\
\hline Gait speed, $\mathrm{m} / \mathrm{s}$ & 1.3 & \pm & 0.2 & 1.3 & \pm & 0.2 & 1.3 & \pm & 0.2 & 0.27 \\
\hline $\mathrm{SMI}, \mathrm{kg} / \mathrm{m}^{2}$ & 6.0 & \pm & 0.9 & 6.1 & \pm & 0.9 & 6.0 & \pm & 0.9 & 0.56 \\
\hline MMSE score & 28.5 & \pm & 2.3 & 28.7 & \pm & 2.1 & 28.0 & \pm & 2.6 & 0.02 \\
\hline GDS- 15 score & 3.3 & \pm & 2.3 & 2.0 & \pm & 1.4 & 6.3 & \pm & 1.4 & 0.00 \\
\hline \multicolumn{11}{|l|}{ Frailty, $n(\%)$} \\
\hline Robust & 152 & (41.9) & & 122 & $(46.7)$ & & 30 & (29.4) & & 0.00 \\
\hline Pre-frail & 185 & $(51.0)$ & & 128 & $(49.0)$ & & 57 & $(55.9)$ & & \\
\hline Frail & 26 & $(7.2)$ & & 11 & $(4.2)$ & & 15 & $(14.7)$ & & \\
\hline \multicolumn{11}{|c|}{ Locomotive syndrome, $n(\%)$} \\
\hline Robust & 97 & (26.8) & & 71 & $(27.3)$ & & 26 & $(25.5)$ & & 0.28 \\
\hline Locomo1 & 160 & (44.1) & & 120 & $(46.0)$ & & 40 & $(39.2)$ & & \\
\hline Locomo2 & 106 & (29.3) & & 70 & (26.9) & & 36 & $(35.3)$ & & \\
\hline \multicolumn{11}{|l|}{ Sarcopenia, $n(\%)$} \\
\hline Robust & 178 & $(49.0)$ & & 128 & $(49.0)$ & & 50 & $(49.0)$ & & 0.97 \\
\hline Pre-sarcopenia & 134 & (36.9) & & 97 & $(37.2)$ & & 37 & $(36.3)$ & & \\
\hline Sarcopenia & 51 & $(14.0)$ & & 36 & (13.8) & & 15 & $(14.7)$ & & \\
\hline
\end{tabular}

SD: standard deviation, SMI: Skeletal Muscle Mass Index,

MMSE: Mini-Mental State Examination, GDS-15: Geriatric Depression Scale

Table 2. Association between depressive symptoms and geriatric syndrome in study participants*

\begin{tabular}{lllcccc}
\hline Frailty & Crude $\mathrm{OR}^{*}$ & $95 \% \mathrm{CI}$ & $p$-value & $\mathrm{aOR}^{\dagger}$ & $95 \% \mathrm{CI}$ & $p$-value \\
Robust & 1.00 & & & 1.00 & & \\
Pre-frail & 1.81 & $1.09-3.01$ & 0.02 & 1.85 & $1.09-3.01$ & 0.02 \\
Frail & 5.55 & $2.31-13.30$ & 0.00 & 5.45 & $2.23-13.31$ & 0.00 \\
\hline & \\
\hline
\end{tabular}


Figures

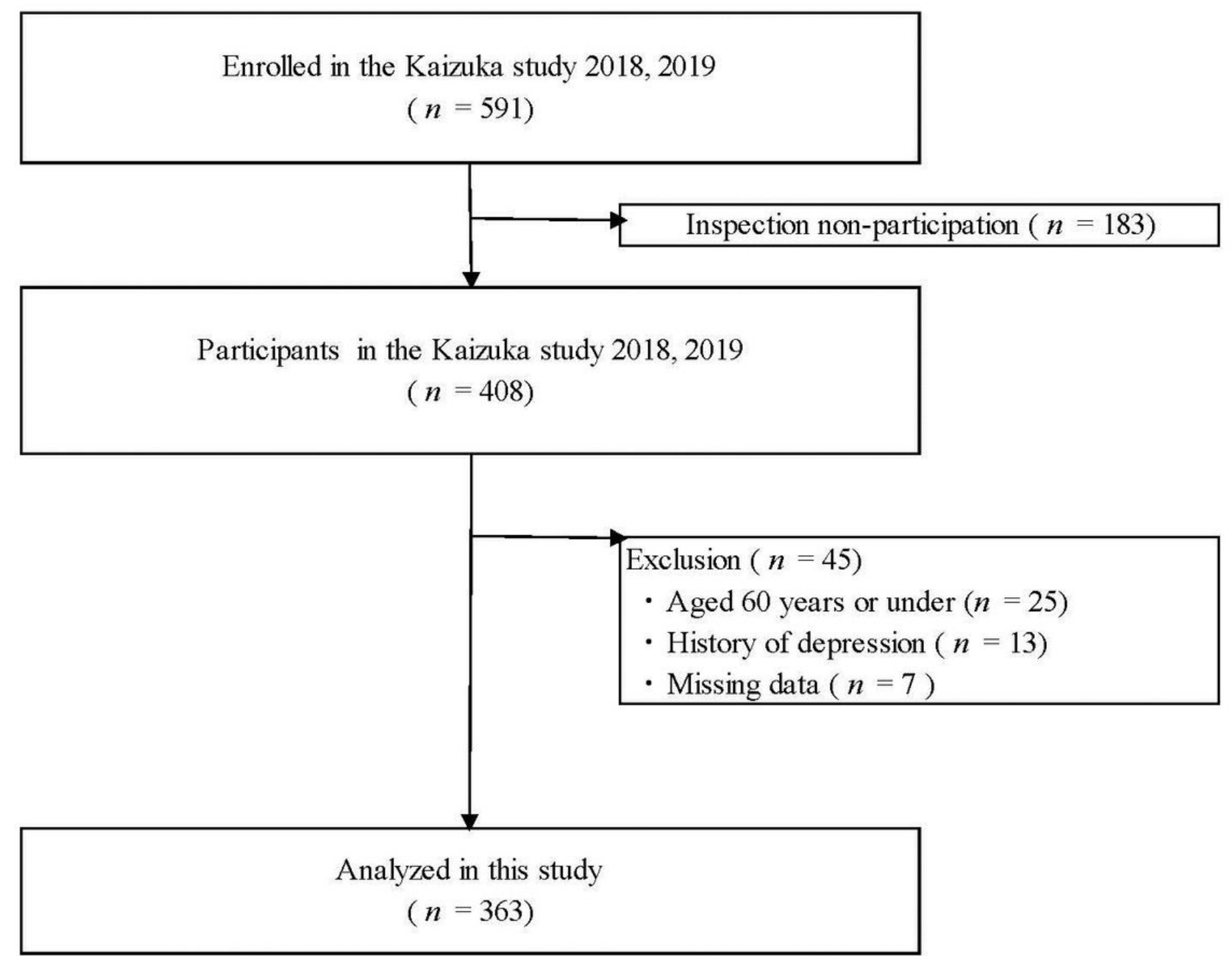

Figure 1

Flow chart for participant recruitment 